\title{
Intégration hémisphérique en l'absence de corps calleux
}

Les deux hémisphères cérébraux constituent des structures autonomes échangeant normalement des informations permettant de coordonner leur fonctionnement grâce au riche faisceau de fibres commissurales que constitue le corps calleux. Si cette structure était la seule à permettre la communication interhémisphérique, les sujets ayant subi une commissurotomie devraient avoir deux cerveaux autonomes traitant éventuellement les mêmes informations de façon contradictoire, avec les conséquences que l'on peut imaginer sur le comportement. En réalité, les structures mésencéphaliques permettent alors une certaine coordination des activités hémisphériques, permettant au cerveau divisé de maintenir une unité d'action et de but.

\section{Justine Sergent}

\section{ADRESSE}

J. Sergent: docteur ès sciences, professeur adjoint au département de neurologie et neurochirurgie de l'université McGill; scientifique du Conseil de la Recherche Médicale du Canada à l'Institut neurologique de Montréal. Institut neurologique de Montréal, 3801 rue Université, Montréal, Québec, H3A 2B4,

e cerveau est composé de deux hémisphères essentiellement symétriques surplombant un tronc commun et reliés entre eux par plusieurs faisceaux de fibres commissurales dont le plus important est le corps calleux. La section du corps calleux et autres commissures néocorticales chez des patients épileptiques réfractaires à toute thérapeutique neurochimique a fourni une occasion unique d'étudier le fonctionnement du cerveau en l'absence de connexions, et donc de moyens de communication directe, entre les deux hémisphères cérébraux (figure 1). Les recherches sur ces patients à cerveau divisé (split brain), initiées par Sperry et ses collaborateurs [1,2], ont confirmé le rôle différent joué par les deux hémisphères dans le contrôle du comportement et des fonctions cognitives et ont démontré que chaque hémisphère pouvait constituer un centre autonome de traitement de l'information, chacun se montrant doué de compétences propres. Il est ainsi apparu, d'une part, que l'hémisphère gauche contribuait de façon exclusive à la production du langage alors que l'hémisphère droit, loin de jouer le rôle mineur qui lui était alors attribué, sous-tendait des fonctions cruciales sous-jacentes à la perception et à la reconnaissance d'objets et possédait certaines capacités de compréhension verbale; d'autre part, la commissurotomie résultait en un syndrome de déconnexion hémisphérique caractérisé par l'impossibilité de communication entre les deux hémisphères et par la coexistence, au sein d'un même cerveau, de deux systèmes cognitifs dont chacun igno- 
rait les opérations et les informations produites par l'autre. Ces conséquences de la section des commissures néocorticales ont été décrites à de multiples reprises et ne seront pas discutées davantage dans cet article [3].

Un autre aspect de la commissurotomie, beaucoup moins frappant mais tout aussi important pour la compréhension du fonctionnement du cerveau divisé, est l'absence de signes de confusion ou de déconnexion dans le comportement des patients en dehors du laboratoire [4, 5]. Sans les expériences spécifiquement conçues pour stimuler séparément les hémisphères cérébraux, on ne disposerait d'aucun indice suggérant la condition particulière de leur cerveau. Il s'agit là d'un paradoxe qui pose un problème fondamental pour la compréhension du fonctionnement du cerveau divisé : malgré la déconnexion et l'isolement de chaque hémisphère, ces patients se comportent comme si leur cerveau était capa-

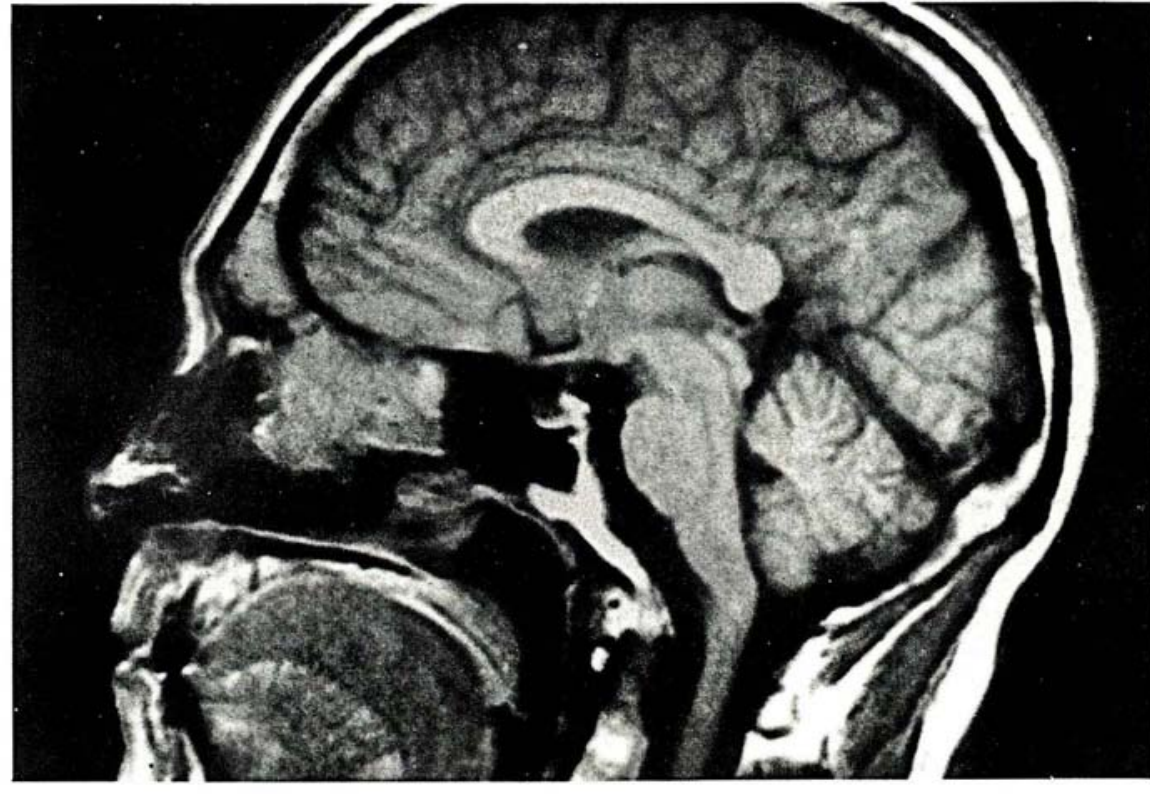

$\Delta$

Figure 1. Coupe transversale du cerveau humain. A : un cerveau intact où le corps calleux (le large faisceau clair) occupe une position centrale à la base des hémisphères; $\boldsymbol{B}$ : un cerveau ayant subi une section complète du corps calleux.

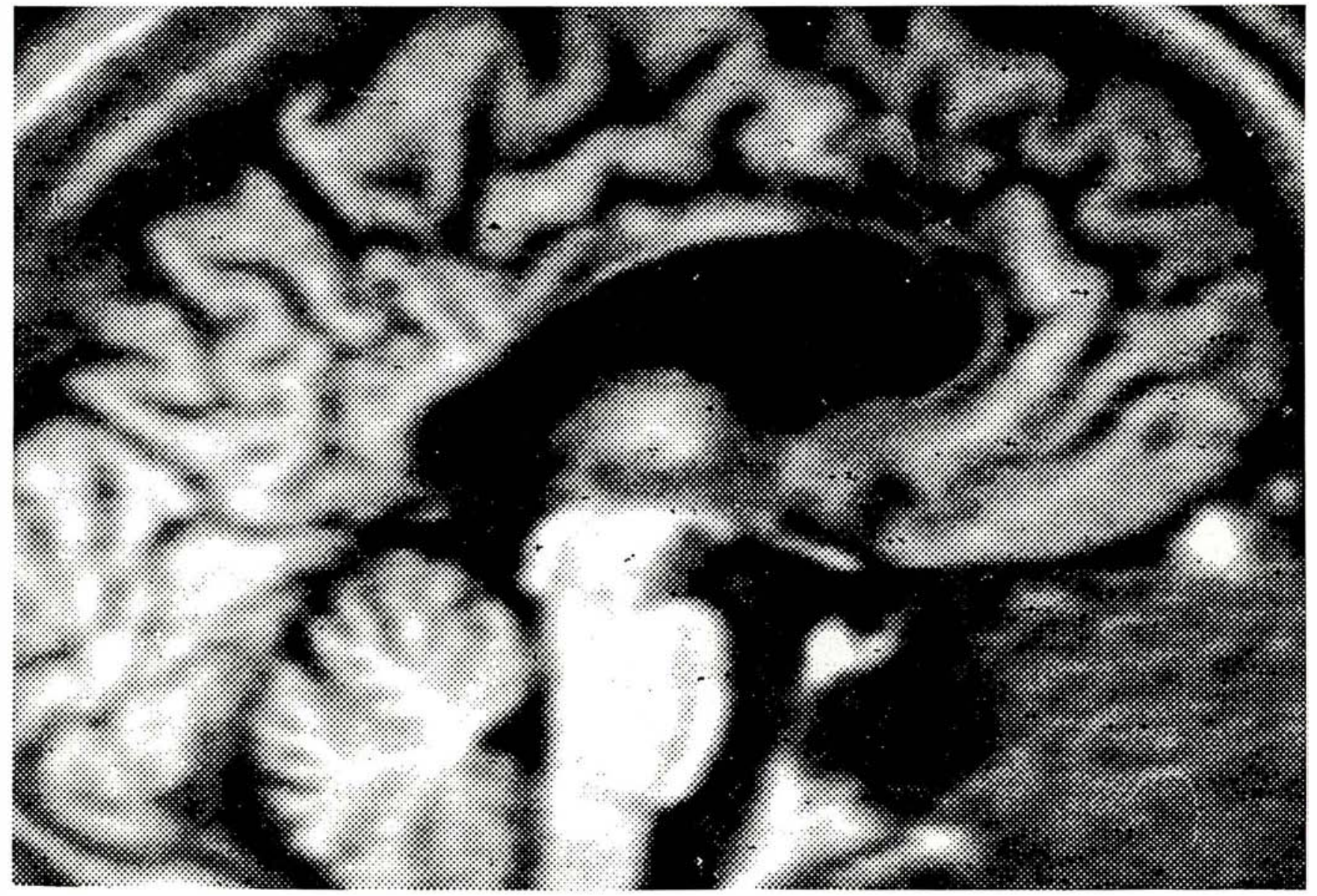


ble d'intégrer les opérations de ses deux hémisphères. Or, si la commissurotomie empêchait toute forme de communication interhémisphérique, ou bien les deux hémisphères se feraient concurrence pour le contrôle du comportement, ce qui se manifesterait par des hésitations ou des confusions, ou bien un hémisphère prendrait le contrôle du comportement, ce qui se traduirait par une négligence du côté ipsilatéral du corps et de l'espace [6]. Ni l'une ni l'autre de ces çonséquences ne sont observées chez les patients commissurotomisés, ce qui suggère que le cerveau divisé possède une certaine capacité à intégrer et à coordonner les ressources de ses hémisphères.

L'existence conjointe d'un syndrome de déconnexion et d'un comportement apparemment normal chez les patients commissurotomisés soulève une série de questions qui feront l'objet de cet article. (1) Pourquoi s'est-on penché presque exclusivement sur la déconnexion hémisphérique dans l'étude de ces patients ? (2) La déconnexion hémisphérique estelle totale? (3) L'apparence de normalité observée hors du laboratoire peut-elle être démontrée dans le laboratoire? (4) Jusqu'à quel point les deux hémisphères déconnectés peuvent-ils coordonner leurs opérations ? (5) Quels sont les mécanismes sous-jacents à l'intégration hémisphérique en l'absence du corps calleux? (6) Quelles en sont les implications pour la compréhension du fonctionnement du cerveau normal ?

\section{Intérêt exclusif pour la déconnexion hémisphérique}

Il serait trop long de décrire ici le contexte dans lequel l'étude des patients commissurotomisés a débuté, mais il est à noter que le corps calleux, plus que toute autre structure cérébrale, a été l'objet de nombreuses spéculations quant à sa fonction réelle, allant du siège de l'âme à un simple support physique empêchant les hémisphères de s'affaisser. Dès 1900, le rôle du corps calleux dans le transfert interhémisphérique avait été suggéré et démontré, entre autres par Déjerine connaissances tombèrent rapidement dans l'oubli de sorte que, dans les années 1940, il était admis que le corps calleux ne jouait pas de rôle important dans le fonctionnement cérébral. D'ailleurs, des expériences menées à cette époque sur des patients épileptiques ayant subi une section du corps calleux n'avaient démontré aucun signe de déconnexion interhémisphérique [9], ce qui avait confirmé l'opinion admise et suscité la boutade selon laquelle le corps calleux ne servait qu'à la propagation des décharges comitiales d'un hémisphère à l'autre. Or, au cours des années 1950, Sperry et Myers [10] démontrèrent de façon irréfutable que la section du corps calleux chez des animaux rendait la communication entre les deux hémisphères impossible, et ils établirent ainsi que la fonction essentielle du corps calleux était le transfert interhémisphérique d'informations. Lorsque la commissurotomie chez des patients épileptiques fut à nouveau effectuée dans les années 1960, il devenait donc important de montrer que les résultats obtenus sur des animaux pouvaient être reproduits chez des humains, et cela contribua à faire de la déconnexion hémisphérique l'objet principal des recherches sur les patients commissurotomisés [11]. Cette priorité donnée à l'étude de la déconnexion semble avoir été accentuée par l'orientation générale de la recherche neuropsychologique : l'absence d'anomalie (le comportement normal du patient dans sa vie quotidienne) ne provoque que peu d'intérêt pour le chercheur alors qu'un déficit (le syndrome de déconnexion) suscite un attrait immédiat. Cette polarisation sur le syndrome de déconnexion eut pour conséquence d'exclure du champ de la recherche la question de la normalité du comportement des patients en dehors du laboratoire. Le choix du qualificatif split brain pour désigner les patients commissurotomisés illustre d'une certaine façon cette orientation. Ce qualificatif n'est pas entièrement adéquat puisqu'il dénote une séparation du cerveau en deux parties alors que seul le cortex se trouve effectivement divisé. Les structures sous-corticales sont en effet épargnées lors de la commissurotomie et constituent, au moins potentiellement, un lieu d'échange entre les deux côtés du cerveau ainsi qu'un tronc commun aux deux hémisphères. Ce choix contestable du qualificatif split brain reflète plus qu'un simple problème sémantique et traduit une orientation conceptuelle qui s'est révélée déterminante dans la façon d'aborder l'étude des patients commissurotomisés, de concevoir la démarche expérimentale et d'interpréter les données obtenues. De fait, en posant comme prémisse la division du cerveau, on prenait pour acquis que les deux hémisphères étaient isolés et indépendants et on écartait ainsi la possibilité qu'ils puissent coordonner leurs opérations. Ceci eut pour conséquence la mise sur pied d'expériences de laboratoire dans lesquelles la communication entre les hémisphères n'était pas requise, ce qui contribuait à perpétuer l'idée que les hémisphères étaient effectivement indépendants. Or, montrer que deux structures peuvent fonctionner isolément n'implique pas qu'elles sont incapables d'intégrer leurs activités, de la même façon que la capacité de faire travailler les deux mains séparément n'implique pas qu'elles sont incapables d'agir de façon coordonnée.

\section{Transmission sous-corticale}

Malgré cette priorité accordée à l'étude du syndrome de déconnexion et du fonctionnement isolé de chaque hémisphère, les recherches se sont néanmoins orientées progressivement vers un examen des capacités de transfert interhémisphérique par l'intermédiaire des structures souscorticales, en utilisant des techniques expérimentales permettant de contrôler l'information projetée à chaque hémisphère. Le système visuel est particulièrement approprié à ce type d'étude puisque chaque champ visuel latéral est représenté dans l'hémisphère controlatéral. Ainsi, en présentant des stimuli dans un champ visuel pendant une durée plus courte que le temps nécessaire au déclenchement d'un mouvement oculaire (environ 180 millisecondes), seul l'hémisphère controlatéral se trouve être stimulé (figure 2). Cette technique s'est révélée très utile à l'étude des patients commissurotomisés et a permis de démontrer 
l'existence du syndrome de déconnexion. Ainsi, lorsque l'on présente simultanément à un patient commissurotomisé deux formes, une dans chaque champ visuel, le patient ne peut ni juger si ces deux formes sont identiques ou non ni nommer la forme apparaissant dans le champ visuel gauche, alors qu'il n'a aucune difficulté à fournir son nom lorsqu'elle est projetée dans le champ visuel droit. Cette technique de stimulation isolée de chaque hémisphère offre donc une méthode pertinente à l'étude de la communication interhémisphérique.

La communication interhémisphérique a ainsi été évaluée en examinant la performance d'un hémisphère en fonction de l'information reçue par l'autre et il est apparu que les patients étaient capables d'indiquer verbalement, et donc par l'intermédiaire de l'hémisphère gauche, la présence et la direction de stimuli se déplaçant dans le champ visuel gauche auquel seul l'hémisphère droit a initialement accès [12]. Des résultats similaires ont été obtenus dans des épreuves portant sur l'orientation de l'attention d'un champ visuel à l'autre [13] et sur certaines informations sémantiques dérivées de la présentation d'un mot dans un champ visuel [14, 15]. Myers et Sperry [16] ont conclu de ces résultats que la transmission sous-corticale se limitait à des informations de nature contextuelle et orientationnelle et que chaque hémisphère demeurait essentiellement ignorant de l'information reçue par l'autre. Ces auteurs ont ainsi suggéré que la transmission interhémisphérique sous-corticale est insuffisante pour compenser la déconnexion hémisphérique et pour sous-tendre l'échange d'informations sans lequel l'intégration des opérations hémisphériques ne peut être effectuée. En d'autres termes, le corps calleux est indispensable au fonctionnement unifié du cerveau.

\section{De l'observation directe au laboratoire}

Si les commissures néocorticales sont indispensables au fonctionnement unifié du cerveau, comment rendre compte de l'apparente normalité du comportement des patients commissurotomisés en dehors du laboratoire? Une réponse à cette question

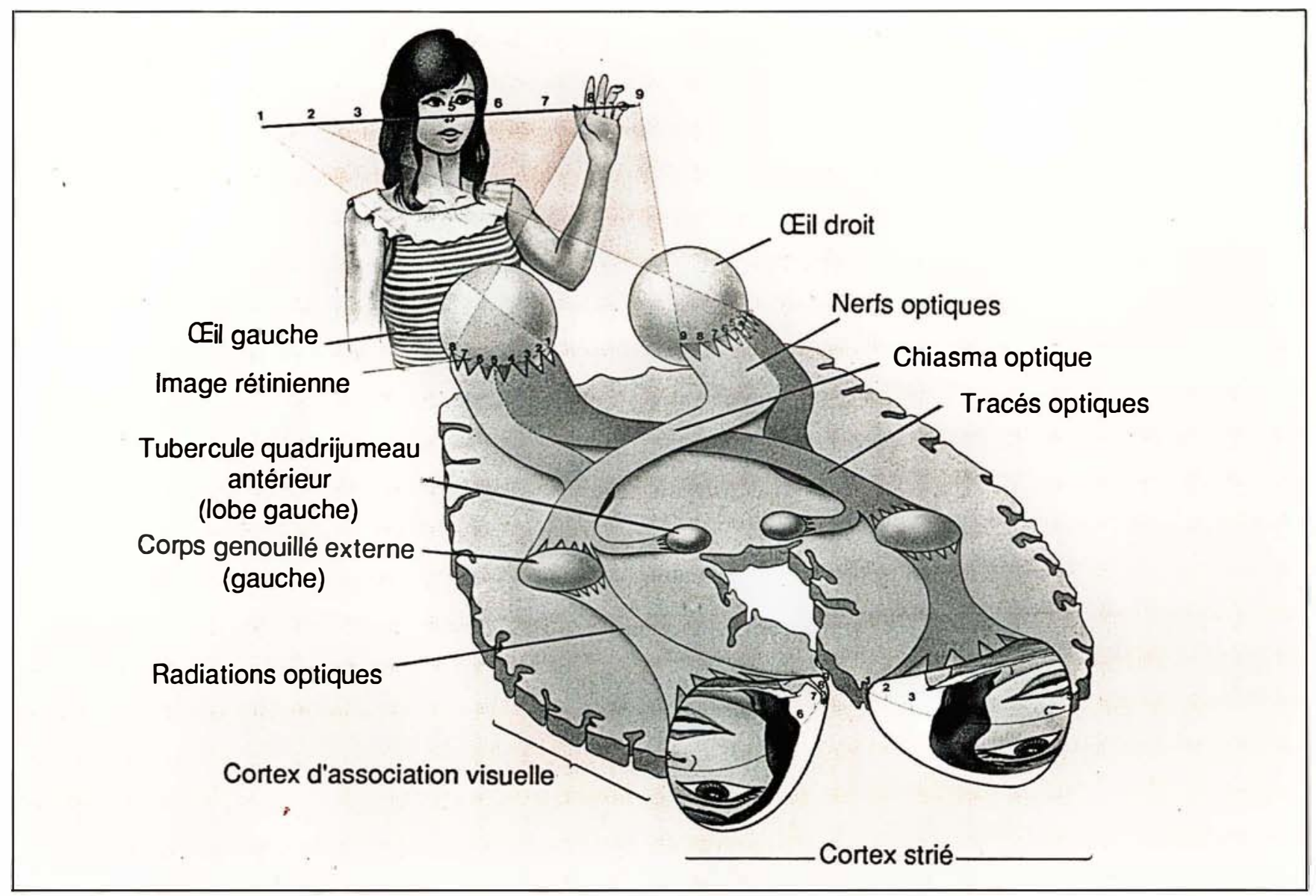

Figure 2. Représentation schématique des voies visuelles allant de la rétine au cortex strié et montrant la projection controlatérale des informations présentées dans un champ visuel vers le cortex (Reproduit de J. Frisby. De l'cil à la vision. Paris: Nathan, 1979). 


\section{RÉFÉRENCES}

1. Sperry RW. Some effects of disconnecting the cerebral hemispheres. Science $1982 ; 217$ : 1223-6.

2. Gazzaniga MS. The bisected brain. New York : Appleton-Century-Crofts, 1970.

3. Brion S, Jedynak CP. Les troubles du transfert interhémisphérique. Paris : Masson, 1975.

4. Sperry RW. Mental unity following surgical disconnection of the cerebral hemispheres. Harvey Lectures 1967 ; 62 : 293-323.

5. Gazzaniga MS, LeDoux J. The integrated mind. New York: Plenum Press, 1978.

6. Joynt RJ. Inattention syndrome in splitbrain man. Adv Neurol 1977 ; 18 : 33-9.

7. Déjerine J. Contribution à l'étude anatomo-pathologique et clinique des différentes variétés de cécité verbale. Comptes rendus des séances et mémoires de la Société de biologie, Paris, 1982 : 61-90.

8. Liepmann H. Drei Aufsätze aus dem Apraxiegebet. Berlin : Karger, 1908.

9. Akelaitis AJ. A study of gnosis, praxis and language following section of the corpus callosum and anterior commissure. J Neurosurg 1945 ; 1 : 94-102.

10. Sperry RW. Cerebral organization and behavior. Science 1961 ; 133 : 1749-57.

11. Sperry MS, Gazzaniga MS, Bogen JE. Interhemispheric relationships : the neocortical commissures; syndromes of hemisphere disconnection. Handbook Clin Neurol 1969 ; $4: 272-90$.

12. Trevarthen C, Sperry RW. Perceptual unity and ambient visual field in human commissurotomy patients. Brain 1973; 96 : 547-70.

13. Holtzman JD, Sidtis JJ, Volpe BT, Wilson DH, Gazzaniga MS. Dissociation of spatial information for stimulus localization and the control of attention. Brain $1981 ; 104$ : 86172.

14. Zaidel E. Reading by the disconnected right hemisphere : an aphasiological perspective. In : Zotterman Y, ed. Dyslexia : neuronal, cognitive and linguistic aspects. Oxford : Per- s'appuie sur le fait que, dans leur environnement normal, les patients ont la possibilité de bouger la tête et les yeux, de sorte que les deux hémisphères reçoivent la même information et partagent la même expérience, ce qui élimine le besoin d'un échange entre les deux hémisphères. Ainsi, l'apparente normalité des patients serait le résultat de mécanismes qui ne requièrent pas une coordination des opérations des hémisphères [17]. Cette explication ne semble pas suffisante cependant, puisqu'il a été démontré que les deux hémisphères pouvaient aboutir à des conclusions différentes après avoir traité la même information [18]. Il est donc nécessaire qu'une certaine coordination soit mise en place pour résoudre les conflits potentiels qui découleraient de décisions différentes prises par chaque hémisphère.

Pour examiner cette question, des patients commissurotomisés ont été testés dans le laboratoire en créant des conditions dans lesquelles les deux hémisphères recevaient simultanément des informations différentes associées à des réponses opposées, et les patients devaient produire une réponse unique sur la base de ces informations en fonction de critères préétablis. Les résultats montrèrent que, dans cette situation, les patients étaient capables de fournir une telle réponse, et que le cerveau commissurotomisé pouvait donc jusqu'à un certain point coordonner les activités de ses deux hémisphères même lorsque ceux-ci recevaient des informations différentes et conflictuelles [19, 20]. En l'absence de commissures néocorticales, une telle coordination ne pouvait s'effectuer qu'à un niveau sous-cortical et ces expériences reproduisaient dans le laboratoire le comportement unifié observé dans l'environnement normal, suggérant que cette normalité n'était pas due à la réception d'informations similaires par les deux hémisphères.

\section{Démonstration d'une intégration hémisphérique}

Les expériences précédentes démontraient la production d'un comportement unifié malgré la déconnexion et la présence d'informations conflictuelles dans les deux hémisphères, mais elles ne permettaient pas de spécifier si cette performance était obtenue par un échange d'informations entre les deux hémisphères ou par une prise de contrôle de la réponse par un seul hémisphère. De fait, les résultats indiquaient une absence de conflits entre les deux hémisphères et c'est donc sur la base de résultats négatifs que l'on concluait à l'existence d'une coordination hémisphérique.

L'étape suivante a donc consisté à chercher des preuves positives d'un échange et d'une coordination interhémisphérique en utilisant un protocole expérimental qui imposait une communication entre les deux côtés du cerveau. Une telle approche fut utilisée par Holtzman et ses collaborateurs [21] dans le cadre de recherches sur la distribution de l'attention dans l'espace visuel. Ils présentèrent dans un champ visuel une grille divisée en neuf cadrans dont l'un contenait un signal indiquant la position où un stimulus serait ensuite présenté dans l'autre champ visuel. Ils trouvèrent que ce signal reçu par un hémisphère facilitait la détection du stimulus reçu par l'autre hémisphère, donnant lieu à des réponses plus rapides qu'en l'absence de signal et qu'en présence d'un signal erroné. Ces auteurs en conclurent que l'absence de corps calleux n'empêchait pas les deux hémisphères d'échanger des informations permettant de diriger l'orientation de l'attention dans l'espace.

Une approche différente fut utilisée par Sergent [22], fondée sur une présentation simultanée d'informations dans chaque champ visuel et conçue de telle sorte que chaque hémisphère recevait seulement une partie des informations nécessaires à la prise de décision. La production d'une réponse correcte n'était possible que si les deux hémisphères parvenaient à relier et à combiner les informations initialement divisées entre eux. Les patients répondaient manuellement plutôt que verbalement, de façon que chaque hémisphère ait la capacité de produire une réponse. Dans une première série d'expériences, les patients devaient établir une relation spatiale entre les informations apparaissant simultanément dans chaque champ visuel (figure 3). Les résultats montrèrent qu'ils répondaient sans hésitation 
et effectuaient ces tâches d'une façon comparable à celle de sujets normaux, n'éprouvant aucune difficulté à établir des relations entre les informations initialement divisées entre les deux hémisphères. Cependant, compte tenu du rôle des structures mésencéphaliques dans certains traitements de l'information spatiale [23], ces résultats - comme ceux obtenus par Holtzman - pouvaient s'expliquer par un traitement de l'information dans ces structures souscorticales, ce qui signifierait que l'intégration ne porte que sur des opérations élémentaires auxquelles la contribution du cortex n'est pas indispensable. Une seconde série d'expériences fut donc entreprise, fondée sur la même procédure mais utilisant des stimuli qui nécessitaient un traitement cortical de l'information [22]. Ainsi les patients devaient additionner deux groupes de points ou deux nombres qui étaient présentés de chaque côté du point de fixation ; ils devaient également effectuer une décision lexicale sur des séries de lettres chevauchant le point de fixation de sorte que chaque hémisphère n'en recevait que la moitié. Les patients furent une nouvelle fois capables d'accomplir ces tâches à un niveau de précision supérieur à la chance, suggérant que les informations divisées entre les deux hémisphères pouvaient être combinées et servir de base à l'émission d'une réponse correcte. Il est important de noter que, dans ces expériences, les informations apparaissant dans un champ visuel étaient insuffisantes pour produire une réponse correcte (figure 3) et que la mise en relation de ces informations était indispensable.

\section{Nature des mécanismes sous-jacents}

Cette intégration interhémisphérique suggérée par la capacité des patients commissurotomisés à répondre de façon unifiée à des informations divisées entre les deux hémisphères peut paraître contredire les résultats solidement établis des recherches sur la déconnexion interhémisphérique. Cette contradiction n'est qu'apparente cependant, comme permettent de l'illustrer certaines caractéristiques des réponses $\mathrm{m} / \mathrm{s} n^{\circ} 10$ vol. 5 , décembre 89

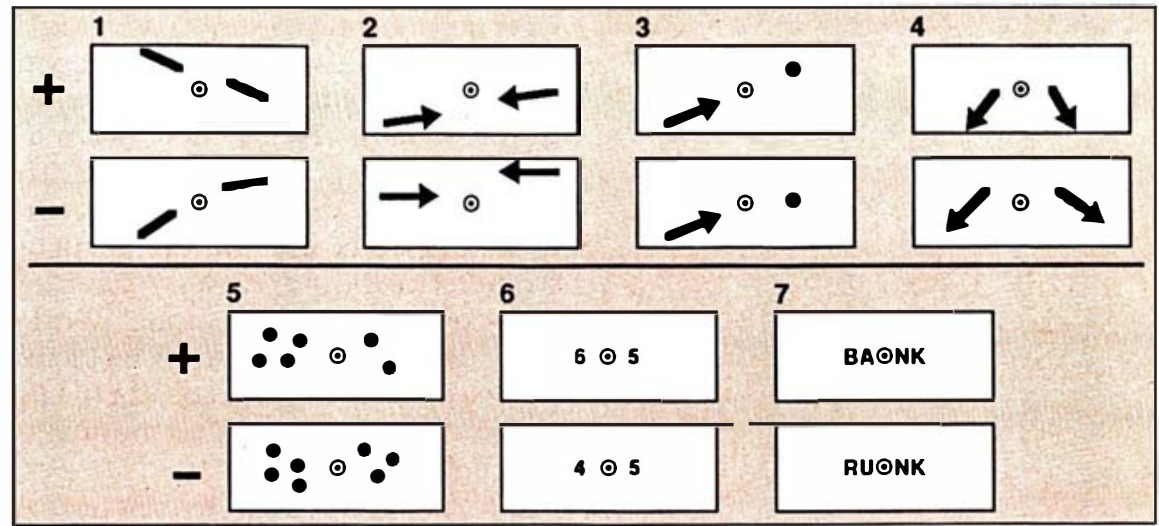

Figure 3. Exemples de stimuli utilisés pour tester l'intégration hémisphérique en I'absence de corps calleux. Alors que les patients fixaient le point central, un stimulus leur était présenté de sorte que chaque hémisphère recevait seulement une moitié des informations nécessaires à la prise de décision. Les patients devaient répondre manuellement en pressant l'un des deux boutons et participèrent à sept expériences différentes comprenant chacune 196 essais. Leur tâche était de décider si: (1) les deux traits forment une ligne droite ou brisée: (2) les flèches se rencontrent ou non; (3) la flèche vise le point ou non; (4) les deux flèches forment un angle inférieur ou supérieur à $90 \%$; (5) la somme des points est paire ou impaire; (6) la somme des chiffres est supérieure ou inférieure aे 10 ; (7) les quatre lettres forment un mot ou non.

des patients dans les expériences décrites précédemment. En effet, malgré leur capacité à émettre une réponse unifiée après la ségrégation de l'information entre les deux hémisphères, les patients se montraient incapables de décrire verbalement les informations apparaissant dans le champ visuel gauche, suggérant que l'hémisphère gauche n'avait pas accès à ces informations (figure 2). L'expérience de décision lexicale offre un exemple frappant de ce phénomène.

Dans cette expérience, quatre lettres étaient présentées et les patients devaient décider si elles formaient ou non un mot. Deux lettres apparaissaient dans le champ visuel gauche et deux lettres dans le champ visuel droit, de sorte qu'aucun hémisphère ne recevait un mot complet (figure 3). Les «non-mots » étaient composés des deux premières lettres d'un vrai mot et des deux dernières lettres d'un autre vrai mot, éliminant tout indice de décision pour chaque hémisphère. Alors que les patients n'avaient pas de difficulté à décider si les quatre lettres formaient un mot ou non, ils étaient incapables de dire quel était le mot et ne parvenaient qu'à nommer les deux lettres apparaissant dans le champ visuel droit et donc projetées dans l'hémisphère gauche. Les deux premières lettres qui apparaissaient dans le champ visuel gauche, et se trouvaient donc projetées dans l'hémisphère droit, ne pouvaient être nommées et demeuraient ainsi en dehors du champ de conscience de l'hémisphère gauche. Cette incapacité des patients à nommer les lettres projetées dans l'hémisphère droit constitue un trait typique du syndrome de déconnexion hémisphérique, et les résultats de ces expériences ne contredisent donc pas les découvertes de Sperry. En revanche, ce que ces expériences démontrent est que cette déconnexion n'empêche pas le cerveau «divisé » d'agir en fonction des informations reçues•par les deux hémisphères déconnectés. Ainsi, même si chaque hémisphère semble ne pas avoir accès aux informations reçues par l'autre et même si les deux hémisphères ne peuvent en partager le contenu à un niveau conscient, ces 


\section{RÉFÉRENCES}

15. Cronin-Golomb A. Subcortical transfer of cognitive information in subjects with complete forebrain commissurotomy. Cortex 1986 ; 22 : 499-519.

16. Myers JJ, Sperry RW. Interhemispheric communication after section of the forebrain commissures. Cortex 1985 ; 21 : 249-60.

17. Sperry RW. Consciousness, personal identity and the divided brain. Neuropsychologia 1984 ; 22 : 661-73.

18. Levy J, Trevarthen C. Metacontrol of hemispheric function in human split-brain patients. Human perception and performance. J Exp Psychol 1976; 2 : 299-312.

19. Sergent J. Unified response to bilateral hemispheric stimulation by a split-brain patient. Nature 1983 ; 305 : 800-2.

20. Sergent J. Subcortical coordination of hemisphere activity in commissurotomized patients. Brain 1986; 109 : 357-69.

21. Holtzman JD. Interactions between cortical and subcortical visual areas: evidence from human commissurotomy patients. Vision Res 1984 ; 24 : 801-13.

22. Sergent J. A new look at the human split brain. Brain 1987; 110 : 1375-92.

23. Tigges J, Tigges M. Subcortical sources of direct projections to visual cortex. In : Peters A, Jones EG, eds. Cerebral Cortex, vol. 3, Visual Cortex. New York: Plenum Press, 1985 : 351-78.

24. Penfield W, Roberts L. Speech and brain mechanisms. Princeton: Princeton University Press, 1959.

25. Fessard AE. Mechanisms of nervous integration and conscious experience. In : Delafresnaye JF, ed. Brain mechanisms and consciousness. Oxford: Blackwell Scientific, 1954: 200-36.

26. Popper K, Eccles JC. The brain and its 752 informations n'en sont pas moins combinées et intégrées d'une façon qui permet l'émission d'une réponse unifiée tenant compte des informations reçues par les deux hémisphères. En l'absence de corps calleux, une telle intégration ne peut être effectuée que par les structures sous-corticales et c'est donc par le biais de ces structures que les deux hémisphères déconnectés peuvent maintenir une certaine communication entre eux.

Dans l'état actuel des recherches, il n'est pas possible de spécifier de façon précise les mécanismes sousjacents à cette intégration ni les structures qui les sous-tendent. Néanmoins, il apparaît que, même si chaque hémisphère demeure ignorant du contenu spécifique des informations initialement reçues par l'autre hémisphère, certains aspects de ces informations convergent vers des structures mésencéphaliques où ils peuvent y être combinés. Alors que la question des processus en jeu demeure essentiellement sans réponse, cette coordination sous-corticale peut donc sous-tendre l'apparente normalité des patients commissurotomisés observée en dehors du laboratoire, offrant la possibilité de mettre en relation les produits respectifs des opérations de chaque hémisphère et de maintenir l'unité d'action et de but qui caractérise le comportement de ces patients dans leurs activités quotidiennes.

\section{Conclusion}

La dualité anatomique du cerveau, par laquelle chaque hémisphère constitue potentiellement une structure autonome, est accompagnée de la présence d'un riche faisceau de fibres commissurales reliant les deux hémisphères et contribuant à la communication interhémisphérique. Bien que le corps calleux soit essentiel à l'échange d'informations spécifiques entre les deux hémisphères et que sa section réduise considérablement la transmission interhémisphérique, il n'est pas indispensable au maintien d'une unité d'action chez les patients commissurotomisés. L'apparente normalité de ces patients dans leur comportement, évidente dès les premiers jours suivant leur opération, met en relief le rôle crucial des structures sous-corticales dans l'intégration des opérations de chaque hémisphère. Même si cette intégration ne porte pas sur toutes les informations reçues ou produites par chaque hémisphère et se révèle insuffisante pour compenser tous les effets de la section du corps calleux, elle n'en est pas moins suffisante pour permettre aux deux hémisphères de coordonner leurs activités et empêcher l'apparition de conflits ou dissociations qui reflèteraient la présence de deux structures entièrement autonomes au sein du même cerveau.

L'étude des patients commissurotomisés confirme donc que les structures mésencéphaliques sont foncièrement actives dans le contrôle du comportement et interagissent avec les structures corticales dans l'élaboration et la réalisation des fonctions cognitives [24, 25]. Les récents résultats anatomiques font état d'un réseau complexe de connexions entre les structures corticales et sous-corticales qui sous-tendent des échanges multiples et permettent une influence réciproque de ces deux structures [23]. De plus, l'étude des patients commissurotomisés permet d'étendre ces connaissances en montrant que les structures mésencéphaliques possèdent les capacités fonctionnelles sous-jacentes à l'intégration des activités corticales dans des opérations cognitives. Cette intégration n'est pas parfaite cependant et ne donne pas nécessairement lieu à une prise de conscience des opérations qui l'accompagnent, ce qui met en relief les limitations de ces structures qui ne peuvent transmettre qu'une partie restreinte des informations produites par chaque hémisphère. Il semble néanmoins que ces structures soient suffisantes pour réaliser une coordination des activités hémisphériques et permettre au cerveau divisé de maintenir une unité d'action et de but. Cela infirme les suggestions [26] voulant que la section du corps calleux crée deux personnes distinctes et indique que, en contradiction avec l'idée d'une dualité mentale chez les patients commissurotomisés, le cerveau cherche à tout prix à maintenir une communication entre ses composantes et donc à préserver l'unité mentale de l'individu 


\section{Summary}

Hemisphere integration in the absence of forebrain commissures Severance of the corpus callosum for the relief of intractable epilepsy results in a hemispheric disconnection syndrome characterized by an inability of the two cerebral hemispheres to communicate between orie another. Yet commissurotomised patients do not display signs of confusion or dissociation in their behaviour, suggesting that the two hemispheres can coordinate their operations in the absence of corpus callosum. When tested in experiments designed to provide each disconnected hemisphere with only part of the information required for a correct response, these patients are able to produce an unified response based on the information received by both hemispheres. This capacity of the commissurotomised brain to combine information initially segregated between the two hemispheres points to a role of the intact subcortical structures in the integration of the activities of the disconnected hemispheres. However, these structures do not take over all communicative functions normally subserved by neocortical commissures. The corpus callosum is required for interhemispheric exchange of specific informational contents, but it is not indispensable for interhemispheric integration which can be subserved by the brain stem, as suggested by the unified behaviour characteristic of commissurotomised patients.

\section{LE GËNE TDF N'EST PAS RESPONSABLE DE LA DÉTERMINATION DU SEXE}

En décembre 1987, le Dr David Page et al. ont isolé ZFY (Zinc Finger Y), gène candidat comme facteur de détermination du sexe (TDF) (m/s no 3, vol. 4, p. 188). Cependant des arguments sont venus compliquer cette adéquation; il existe sur le chromosome $X$ un gène homologue ZFX, qui échappe au phénomène d'inactivation. Enfin, et surtout, l'équivalent du gène ZFY n'est plus, chez les marsupiaux, porté par le chromosome $Y$ mais par un autosome, bien que la détermination du sexe dans cette espèce suive les mêmes règles que chez les mammifères.

En décembre 1989, deux groupes de généticiens animés, I'un à ICRF par le Dr Peter Goodfellow, l'autre à l'institut Pasteur (INSERM U.276) par le Dr Marc Fellous, ont démontré définitivement que le gène de détermination du sexe, TDF, ne peut pas être ZFY. Ces recherches qui vont faire l'objet d'une publication dans le numéro du 15 décembre 1989 de Nature [1] reposent sur l'étude de cas sporadiques ou familiaux de mâles $X X$ et $d$ 'hermaphrodites ne possédant jusqu'ici aucun matériel génétique du chromosome $Y$. L'identification de ce syndrome rare n'a d'ailleurs pu être faite que grâce à une patiente et fructueuse collaboration avec des pédiatres français et algériens (Dr C. Fekete-Nihoul, C. Turc-Carel, R. Rappaport, J. Toublanc, C. Boucekkine).

Nous avons pu démontrer que quatre patients étaient porteurs d'un très petit fragment d'ADN de 30 à $60 \mathrm{~kb}$, issu de la région frontière du chromosome $Y$, proche de la partie pseudoautosomique. Or ce petit fragment d'ADN ne contient pas la région du gène ZFY!

Ces résultats relancent donc la course désespérée au gène TDF que I'on sait contenu dans ces 30 à $60 \mathrm{~kb}$ d'ADN !

Cependant, nos découvertes posent certaines questions :

- Pourquoi un gène aussi important en reproduction est-il si proche de la région pseudo-autosomique $X Y$ ?

- Pourquoi dans une même famille porteuse de cette petite région d'ADN du chromosome $Y$, un enfant est-il $X X$ mâle tandis que l'autre est hermaphrodite avec ovotestis ?

- Pourquoi par une élégante approche de la génétique dite inverse, le Dr David Page et al. se sont-ils trompés dans la localisation de TDF? Y a-t-il eu inactivation de ce gène par effet de position chez une femme $X Y$ qu'il décrit et qui possède cette région d'ADN?

L'analyse de ces 30 à $60 \mathrm{~kb}$ d'ADN, malheureusement très riches en séquences d'ADN répété, devrait donc dans les prochains mois nous permettre de répondre à ces passionnantes questions.

1. Palmer MS, Sinclair AH, Berta P, Goodfellow PN, Ellis NA, Abbas NE, Fellous $M$. Genetic evidence that ZFY is not the testis determining factor. Nature Décembre 1989 (sous presse). 